Service social

\title{
Anatomie de la prison, par Guy Lemire, Montréal, Presses de l'Université de Montréal (PUM), coll. Criminologie, 195 pages.
}

\section{Germain Trottier}

Volume 40, numéro 1, 1991

Éthique et intervention sociale

URI : https://id.erudit.org/iderudit/706520ar

DOI : https://doi.org/10.7202/706520ar

Aller au sommaire du numéro

Éditeur(s)

École de service social de l'Université Laval

ISSN

1708-1734 (numérique)

Découvrir la revue

Citer ce compte rendu

Trottier, G. (1991). Compte rendu de [Anatomie de la prison, par Guy Lemire, Montréal, Presses de l'Université de Montréal (PUM), coll. Criminologie, 195 pages.] Service social, 40(1), 145-146. https://doi.org/10.7202/706520ar d'utilisation que vous pouvez consulter en ligne.

https://apropos.erudit.org/fr/usagers/politique-dutilisation/ 
la pratique de travail social en a été lourdement touchée. Elle devient malheureusement axée davantage sur "l'application " de politique administrative locale sur l'intervention individuelle que sur la créativité, l'animation et la coordination. Par contre, profitant d'une période de flou, certains travailleurs sociaux ont su profiter de la décentralisation pour s'organiser localement, se doter d'objectifs précis à partir d'une évaluation rigoureuse de la situation. L'auteur conclut que la décentralisation n'est pas nécessairement la source de plus de participation et de créativité et qu'il faut plutôt compter sur le dynamisme des intervenants.

Se référant au concept d'innovation de Michel Crozier, A. Bouconge décrit les principales difficultés d'un véritable changement social durable.

Pierre Coulmin situe les forces du développement local sur les capacités à s'organiser en mouvement social, alors que Pierre Valarié les situe surtout sur le plan de la gestion.

Guy Raffi, pour sa part, s'interroge sur les conditions vraies de la création sociale, en dehors de l'expérience unique difficilement transférable et généralisable. Il conclut sur une note mi-pessimiste, mi-optimiste qu'en dépit du fait que "les institutions ne sont pas capables de créativité, qu'il y a peu de pratiques ou de produits nouveaux et que le travail social n'est pas en liberté ", le monde change.

En somme, comme Michel Kaiser, l'ensemble des auteurs expliquent que le défi est lancé puisque :

"Les multiples crises économiques, sociales, financières qui traversent aujourd'hui la vie sociale mettent au défi les politiques d'action sociale d'y inventer, d'y mobiliser et d'y composer les réseaux de solidarité qui en forment les trames locales. "

Ce défi nous concerne aussi, à nous de le relever.

Nicole Boucher

École de service social

Université Laval

Anatomie de la prison, par Guy LEMIRE, Montréal, Presses de I'Université de Montréal (PUM), coll. Criminologie, 195 pages.

L'ouvrage, constitué de huit chapitres sensiblement égaux en longueur, examine le milieu carcéral pour hommes en y effectuant une véritable autopsie qui démontre l'à-propos de son intitulé.

L'auteur, fort de son expérience professionnelle en centre pénitentiaire et d'enseignant universitaire, introduit le lecteur dans l'univers de l'incarcération en abordant tour à tour des aspects qui concernent fondamentalement l'existence et la coexistence en prison.

Dans un style littéraire sobre, l'auteur nous familiarise avec la quotidienneté des règles formelles et informelles qui tissent les rapports sociaux et contribuent à développer la sous-culture du milieu. II décrit les enjeux antagonistes des acteurs principaux (prisonniers, gardiens, directeurs) et explique ce qui les rend complémentaires dans la dynamique institutionnelle. 
Puisant dans les écrits et les travaux de recherche sur la prison, l'auteur du livre démontre comment la synergie de complaisance parvient à régulariser les transactions, apaiser la violence potentielle et rendre les rapports quasi harmonieux entre individus aux intérêts si divergents en apparence. Ses réponses sont bien articulées et il maîtrise l'univers qu'il décrit. Avec pertinence, il reconnaît le bien-fondé du mouvement de la démocratie en prison, mais il s'interroge aussi sur les obstacles que la situation risque d'entraîner.

Ce livre, initialement destiné aux intervenants et intervenantes en milieu correctionnel, a le mérite d'analyser le milieu carcéral sans prendre parti pour un des protagonistes. En traçant un portrait fidèle et réaliste de la situation, l'auteur décrit sans juger, expose sans imposer, explique sans endoctriner. II est soucieux de présenter des faits manifestes à son lecteur pour l'aider à comprendre les caractéristiques contraignantes d'un milieu carcéral et les gens qui s'y côtoient : gestionnaires, professionnels, gardiens et prisonniers.

La recommandation d'achat de ce volume convient donc à une grande diversité de personnes, mais particulièrement aux praticiens et praticiennes actuels et futurs des milieux de détention. Il est un ouvrage d'intérêt pour quiconque s'intéresse à la problématique de la criminalité adulte, même sans être directement impliqué dans le milieu.

La structure peu complexe du volume ainsi que le style d'écriture limpide et sans artifice rendent sa lecture facile et agréable. L'argumentation, qui évite les débats idéologiques compliqués, se centre sur l'essentiel et s'appuie sur des données éprouvées par les connaissances et l'expérience. Le fil conducteur de l'ouvrage se retrace logiquement et sans ambiguïté.

Pour terminer, nous formulons deux commentaires moins positifs sur des aspects qui viennent affaiblir quelque peu la qualité de l'ouvrage : une conclusion trop courte qui met fin abruptement à la démarche d'analyse poursuivie et aussi des sources documentaires principales qui commencent à dater. Dans le premier cas, l'auteur donne l'impression d'avoir manqué de souffle pour boucler son ouvrage; dans le second, les références donnent à penser qu'il n'y avait ni plus ni mieux dans la littérature toute récente sur le sujet. Dans les deux cas les impressions auraient pu être évitées compte tenu de la qualité générale du livre et de l'intérêt populaire qu'il pourra susciter.

Germain Trottier

École de service social

Université Laval

\section{La relation d'aide : éléments de base et guide de perfection- nement ( $3^{e}$ éd.), par Jean-Luc HÉTU, Boucherville, Gaëtan Morin, éditeur, 1990, 181 pages.}

Depuis sa première édition en 1982, le livre de Jean-Luc Hétu sur la relation d'aide est devenu une sorte de "classique " dans les universités et collèges du Québec. En 1990, ce manuel en est à sa troisième édition et constituera sans doute encore un outil très utilisé.

Le livre débute par un essai de définition du concept d'aide. L'auteur considère qu'une relation est aidante " lorsqu'elle facilite l'accès aux informations subjectives que sont les sentiments, et qu'elle permet au sujet de clarifier 